\title{
Modeling of the Heat Capacity of Polymers with the Variable Connectivity Index
}

\author{
Dahuan LIU and Chongli ZHONG ${ }^{\dagger}$ \\ Department of Chemical Engineering, PO Box 100, Beijing University of Chemical Technology, \\ Beijing 100029, China
}

(Received September 9, 2002; Accepted October 12, 2002)

\begin{abstract}
Correlations for the heat capacity at constant pressure of solid and liquid polymers at room temperature were proposed based on the variable connectivity index in this work. The experimental heat capacity data of 81 solid polymers and 79 liquid polymers were used to establish the correlations, and the average absolute deviations of the heat capacity are $4.8 \%$ and $3.8 \%$, respectively. The correlations proposed are simple, which can be used to predict the heat capacity of polymers at room temperature as long as the chemical structure of the polymer concerned is known.

KEY WORDS Modeling / Heat Capacity / Polymer / Connectivity Index /
\end{abstract}

The heat capacity is a macroscopic, thermodynamic quantity that is based on the molecular motion, which is one of the most important thermophysical properties of materials. It can be used to calculate other calorimetric properties, such as the enthalpy, entropy, and Gibbs free energy, which is also useful in estimating the thermal conductivity. Since most polymer manufacture and processing involve heat transfer, the knowledge of the heat capacity is very important in polymer processing operations.

A lot of experimental heat capacity data have been measured, but its accurate measurement is still difficult, and the data may show large discrepancy even for some well-characterized polymers. Wunderlich et al. analyzed all available data for molar heat capacity at constant pressure, $C_{\mathrm{p}}(T)$, and determined and listed extensive tables of smoothed values of $C_{\mathrm{p}}(T)$, which contributed greatly to progress in this area. ${ }^{1}$

Theoretical models have also been proposed, ${ }^{1-5}$ and one of the commonly used methods for the estimation of the heat capacity at constant pressure at room temperature $\left(C_{\mathrm{p}}(298 \mathrm{~K})\right)$ is the group contribution methods proposed by Satoh and Shaw. ${ }^{2}$ Recently, connectivity indices, the widely used structure descriptors for normal molecules, have been applied to establish predictive models for the properties of polymers, and correlations have been proposed successfully for a wide variety of properties. ${ }^{1}$ More recently, an approach was proposed in our previous work ${ }^{6}$ to calculate high order connectivity indices for polymers in a consistent manner.

The previous investigations ${ }^{1,6,7}$ show that connectivity indices are quite useful in developing simple correlations for the prediction of the properties of polymer and polymer solutions from molecular structure infor- mation. In this work, the variable connectivity index proposed by Randic ${ }^{8}$ is used to establish correlations for the $C_{\mathrm{p}}(298 \mathrm{~K})$ of polymers and compared with those correlations based on the traditional connectivity index.

\section{THEORY}

\section{The Connectivity Index}

Connectivity indices have been widely used as structure descriptors, which contain a large amount of information about the molecule, including the numbers of hydrogen and non-hydrogen atoms bonded to each non-hydrogen atom, the details of the electronic structure of each atom, and the molecular structural features (paths, branches, clusters, and rings). ${ }^{1,9,10}$ Details of their definition and the calculation method can be found elsewhere, ${ }^{9,10}$ and a review on the development of the connectivity index was recently published by Randić. ${ }^{11}$

The general expression for the mth-order connectivity index is as follows:

$$
{ }^{m} \chi_{t}=\sum_{j=1}^{n_{m}} \prod_{i=1}^{m+1}\left(\delta_{i}\right)_{j}^{-0.5}
$$

where $m$ is the order of the connectivity index, $t$ denotes a contiguous path type of fragment, which is divided into paths $(\mathrm{P})$, clusters $(\mathrm{C})$, path/clusters (PC), and chains (cycles) $(\mathrm{CH}) . n_{m}$ is the number of the relevant paths, and $\delta_{i}$ is the simple connectivity index, equal to the number of non-hydrogen atoms to which the ith non-hydrogen atom is bonded.

If $\delta_{i}$ is replaced by $\delta_{i}^{v}$, the valence connectivity index, we can obtain the expression for the mth-order valence connectivity index, ${ }^{m} \chi_{t}^{v}$ as follows:

${ }^{\dagger}$ To whom correspondence should be addressed (Tel: +86-10-64419862; Fax: +86-10-64436781; E-mail: zhongcl@mail.buct.edu.cn). 


$$
{ }^{m} \chi_{t}^{v}=\sum_{j=1}^{n_{m}} \prod_{i=1}^{m+1}\left(\delta_{i}^{v}\right)_{j}^{-0.5}
$$

Recently, a new kind of connectivity index has been introduced, ${ }^{8}$ that is the so-called variable connectivity index, which can be calculated by the following expression:

$$
{ }^{m} \chi_{t}^{f}=\sum_{j=1}^{n_{m}} \prod_{i=1}^{m+1}\left(\delta_{i}^{f}\right)_{j}^{-0.5}
$$

where the variable connectivity index, $\delta_{i}^{f}$, is related to the simple connectivity index, $\delta_{i}$, by

$$
\delta_{i}^{f}=\delta_{i}+x_{i}
$$

where $x_{i}$ is a variable for a non-hydrogen atom, and the numerical value for the variable needs to be selected so to minimize the standard error for a regression. Therefore, $x_{i}$ is a weight variable, which can increase or decrease the contribution of the atom to the property concerned. As a result, the numerical value of $x_{i}$ for an atom may vary largely for different properties, and its value for the same atom in cyclic structure and acyclic structure may be different. ${ }^{12}$ The main advantage of the variable connectivity index is that it gives flexibility to connectivity index as structure descriptor, and therefore can increase correlative accuracy with a simpler expression. However, the numerical values of $x_{i}$ should be regressed by fitting experimental data, and they may change largely when another property is concerned. On the other hand, for a certain property once the numerical values of the weights are determined, the variable connectivity indices can be calculated as simple as the traditional connectivity indices. Therefore, the correlations based on the variable connectivity index have the same predictive capability as those based the traditional connectivity index.

The Existing Correlations for $C_{p}(298 K)$ of Polymers Based on the Traditional Connectivity Index

Based on connectivity index, Bicerano ${ }^{1}$ has proposed two correlations for $C_{\mathrm{p}}(298 \mathrm{~K})$ of solid and liquid polymers separately as follows:

$$
\begin{aligned}
C_{\mathrm{p}}^{\mathrm{S}}(298 \mathrm{~K}) & =8.985304{ }^{0} \chi+20.920972{ }^{1} \chi^{v} \\
& +7.304602\left(N_{\text {rot }}+5 N_{\mathrm{Si}}\right) \\
C_{\mathrm{p}}^{\mathrm{l}}(298 \mathrm{~K}) & =8.162601{ }^{0} \chi+23.215188{ }^{0} \chi^{v} \\
& +8.477370 N_{\mathrm{BBrot}}+5.350331 N_{\mathrm{SGrot}}
\end{aligned}
$$

where $C_{\mathrm{p}}^{\mathrm{S}}(298 \mathrm{~K})$ and $C_{\mathrm{p}}^{\mathrm{l}}(298 \mathrm{~K})$ are the heat capacity at constant pressure at room temperature for solid and liquid polymers, respectively. $N_{\text {BBrot }}$ and $N_{\text {SGrot }}$ are the rotational degrees of freedom of the backbone and side group portions of the repeat units of polymers, respectively. $N_{\text {rot }}$ is the total number of rotational degrees of freedom, and $N_{\mathrm{si}}$ is the number of silicon atoms.

The heat capacity of polymers at a certain temperature can be estimated by the following two expressions: ${ }^{1}$

$$
C_{\mathrm{p}}^{\mathrm{S}}(T)=C_{\mathrm{p}}^{\mathrm{S}}(298 \mathrm{~K})(0.106+0.003 T)
$$

and

$$
C_{\mathrm{p}}^{\mathrm{l}}(T)=C_{\mathrm{p}}^{\mathrm{l}}(298 \mathrm{~K})(0.613+0.0013 T)
$$

As a result, as long as the $C_{\mathrm{p}}(298 \mathrm{~K})$ is known, the $C_{\mathrm{p}}(T)$ at the temperature interest can be estimated using eqs 6 and 7 .

Another important heat capacity value is the change in the heat capacity at the glass transition, $\Delta C_{\mathrm{p}}\left(T_{\mathrm{g}}\right)$. Obviously, it can be calculated readily using eqs 6 and 7 , supposed the required $T_{\mathrm{g}}$ is known for a polymer.

\section{Development of New Correlations Based on the Vari-} able Connectivity Index

The variable connectivity index is more flexible in describing the chemical structure of molecules, which has been successfully applied to a variety of properties of normal molecules. ${ }^{10}$ In this work, we will adopt it to model the physical properties of polymers, and correlations based on the variable connectivity index will be developed for $C_{\mathrm{p}}^{\mathrm{S}}(298 \mathrm{~K})$ and $C_{\mathrm{p}}^{\mathrm{l}}(298 \mathrm{~K})$ in the following section.

It should be pointed out that Bicerano ${ }^{1}$ has tried to optimize the value of $\delta^{\mathrm{V}}$ for each distinct electronic configuration of each atom, for each property, and thus to establish simpler correlations, and applied this method to the correlation of the van der Waals volume of polymers as an example. The basic idea of Bicerano's method is the same as that the variable connectivity index method proposed by Randić. ${ }^{8}$

\section{RESULTS AND DISCUSSION}

\section{Correlation of $C_{p}^{S}$ (298K) for Solid Polymers}

The experimental data of $C_{\mathrm{p}}^{\mathrm{S}}(298 \mathrm{~K})$ for solid polymers collected by Birecano ${ }^{1}$ from the literature were used as the database to establish the new correlation for $C_{\mathrm{p}}^{\mathrm{S}}(298 \mathrm{~K})$. The experimental data and the variable connectivity index used are shown in Table I. Based on these data, the following correlation was obtained:

$$
C_{\mathrm{p}}^{\mathrm{S}}(298 \mathrm{~K})=52.287^{0} \chi^{f}-3.9356
$$

The optimal weights for the non-hydrogen atoms in the calculation of ${ }^{0} \chi{ }^{f}$ were obtained by fitting the data, 
Table I. Calculated results for $C_{\mathrm{P}}^{\mathrm{S}}(298 \mathrm{~K})$ of solid polymers and the variable connectivity indices used

\begin{tabular}{|c|c|c|c|c|}
\hline \multirow[t]{2}{*}{ Polymer } & \multirow[t]{2}{*}{${ }^{0} \chi^{f}$} & \multicolumn{3}{|c|}{$\begin{array}{c}C_{\mathrm{P}}^{\mathrm{S}}(298 \mathrm{~K}) \\
\left(\mathrm{J} \mathrm{mol}^{-1} \mathrm{~K}^{-1}\right)\end{array}$} \\
\hline & & exp. & this work & $\overline{\text { Bicerano }}$ \\
\hline Poly(p-phenylene $)$ & 1.7171 & 84.5 & 85.8 & 86.4 \\
\hline $\operatorname{Poly}(\operatorname{oxy}(p$-phenylene $))$ & 1.9691 & 108.5 & 99.0 & 103.4 \\
\hline Poly(thio $(p$-phenylene $))$ & 2.4314 & 110.2 & 123.2 & 120.5 \\
\hline Poly( $p$-hydroxybenzoate) & 2.6404 & 121.8 & 134.1 & 134.4 \\
\hline Polystyrene & 2.5904 & 126.5 & 131.5 & 133.5 \\
\hline Poly( $p$-xylylene) & 2.6188 & 134.7 & 133.0 & 133.8 \\
\hline Poly ( $p$-chloro styrene) & 2.9673 & 141.1 & 151.2 & 151.3 \\
\hline Poly ( $p$-bromo styrene) & 2.8936 & 144.9 & 147.4 & 160 \\
\hline $\operatorname{Poly}(\alpha$-methyl styrene $)$ & 3.0646 & 149.8 & 156.3 & 156.5 \\
\hline Poly( $p$-methyl styrene) & 3.084 & 150.0 & 157.3 & 157.2 \\
\hline Poly( $N$-vinyl pyrrolidone) & 2.8099 & 158.9 & 143.0 & 156.5 \\
\hline Poly(vinyl benzoate) & 3.5138 & 162.4 & 179.8 & 181.3 \\
\hline Polyoxynaphthoate & 3.7775 & 180.6 & 193.6 & 186.8 \\
\hline $\operatorname{poly}(\alpha$-vinyl naphthalene $)$ & 3.7275 & 192.9 & 191.0 & 186.1 \\
\hline Poly(vinyl butyral) & 4.0522 & 204.6 & 207.9 & 208.8 \\
\hline Poly(vinyl $p$-ethylbenzoate) & 4.4582 & 217.6 & 229.2 & 230.4 \\
\hline Poly(vinyl $p$-isopropylbenzoate) & 4.9234 & 235.4 & 253.5 & 253.5 \\
\hline $\operatorname{Poly}(N$-vinyl carbazole $)$ & 4.8352 & 245.8 & 248.9 & 223.8 \\
\hline Bisphenol-A polycarbonate & 5.9998 & 304.9 & 309.8 & 308.3 \\
\hline Poly(ether ether ketone) (PEEK) & 6.3266 & 319.7 & 326.9 & 324.2 \\
\hline $\operatorname{Poly}\left(N, N^{\prime}\right.$-( $p, p^{\prime}$-oxydiphenylene)pyromellitimide $)$ & 8.6143 & 420.6 & 446.5 & 393.1 \\
\hline Udel & 9.7859 & 491.2 & 507.7 & 498.0 \\
\hline Ultem & 13.691 & 720.5 & 711.9 & 653.5 \\
\hline Polyethylene & 0.9017 & 43.4 & 43.2 & 48.2 \\
\hline Polypropylene & 1.3669 & 67.8 & 67.5 & 71.6 \\
\hline Poly(1,4-butadiene) & 1.8033 & 88.0 & 90.4 & 81.8 \\
\hline Polyisobutylene & 1.8411 & 94.0 & 92.3 & 93.7 \\
\hline Polyisoprene & 2.2686 & 108.0 & 114.7 & 105.4 \\
\hline Poly(4-methyl-1-pentene) & 2.7338 & 144.5 & 139.0 & 143.2 \\
\hline Polyoxymethylene & 0.7028 & 38.3 & 32.8 & 39.4 \\
\hline Polyoxyethylene & 1.1536 & 55.1 & 56.4 & 63.5 \\
\hline Poly(vinyl chloride) & 1.2502 & 59.0 & 61.4 & 65.9 \\
\hline Poly(vinyl fluoride) & 1.1411 & 59.5 & 55.7 & 56.8 \\
\hline Poly(glycolic acid) & 1.3742 & 65.1 & 67.9 & 70.8 \\
\hline Polyacrylonitrile & 1.6645 & 68.4 & 83.1 & 69.3 \\
\hline Polyoxytrimethylene & 1.6045 & 79.3 & 80.0 & 87.6 \\
\hline Poly(vinylidene chloride) & 1.6077 & 80.9 & 80.1 & 81.9 \\
\hline Polytrifluoroethylene & 1.629 & 87.7 & 81.2 & 75.8 \\
\hline Polytetrafluoroethylene & 1.8774 & 89.8 & 94.2 & 85.8 \\
\hline Polychlorotrifluoroethylene & 1.9865 & 99.6 & 99.9 & 93.7 \\
\hline Poly(vinyl acetate) & 2.2903 & 101.2 & 115.8 & 118.6 \\
\hline Poly(methylacrylate) & 2.2903 & 115.0 & 115.8 & 117.8 \\
\hline $\operatorname{Poly}(\alpha$-methyl acrylamide) & 2.3592 & 118.1 & 119.4 & 120.3 \\
\hline Poly(menthyl methacrylate) & 2.7645 & 137.0 & 140.6 & 140.7 \\
\hline Poly( $\varepsilon$-caprolactone $)$ & 3.1775 & 161.6 & 162.2 & 167.2 \\
\hline Poly(ethyl methacrylate) & 3.2153 & 166.5 & 164.2 & 166.7 \\
\hline $\operatorname{Poly}(\varepsilon$-caprolactam $)$ & 3.2574 & 168.9 & 166.4 & 169.6 \\
\hline $\operatorname{Poly}(n$-butyl acrylate) & 3.6428 & 210.0 & 186.5 & 192 \\
\hline Poly(n-butyl methacrylate) & 4.117 & 235.9 & 211.3 & 214.9 \\
\hline Poly(vinyl alcohol) & 1.1222 & 67.4 & 54.7 & 64.9 \\
\hline Poly(methacrylic acid) & 2.2678 & 111.9 & 114.6 & 118.9 \\
\hline Poly(propylene sulfone) & 2.3902 & 123.3 & 121.0 & 119.7 \\
\hline Poly(ethylene oxalate) & 2.7484 & 129.1 & 139.8 & 142.4 \\
\hline Poly(1-butene sulfone) & 2.3869 & 146.8 & 120.9 & 144.7 \\
\hline
\end{tabular}




\begin{tabular}{|c|c|c|c|c|}
\hline Poly(oxy(2,6-dimethyl-1,4-phenylene)) & 2.9563 & 149.0 & 150.6 & 151.1 \\
\hline Poly(oxy(2,6-dimethyl-5-bromo-1,4-phenylene) & 3.2594 & 169.0 & 166.5 & 177.8 \\
\hline Poly(vinyl trimethylsilane) & 3.2409 & 179.1 & 165.5 & 177.8 \\
\hline Poly(dimethyl itaconate) & 4.1387 & 207.6 & 212.5 & 210.9 \\
\hline Poly(1-hexene sulfone) & 3.7427 & 209.5 & 191.8 & 192.9 \\
\hline Poly(isobutyl methacrylate) & 4.1314 & 222.1 & 212.1 & 213.4 \\
\hline Poly(ethylene terephthalate) & 4.4655 & 223.9 & 229.6 & 228.9 \\
\hline Poly(vinyl dimethylphenylsilane) & 4.4644 & 231.0 & 229.5 & 240.6 \\
\hline $\operatorname{Poly}(p$-methacryloxy benzoic acid) & 4.9083 & 260.2 & 252.7 & 253.3 \\
\hline Poly(tetramethylene terephthalate) & 5.3671 & 267.9 & 276.7 & 277.1 \\
\hline Poly(ethylene-2,6-naphthalenedicarboxylate) & 5.6025 & 268.5 & 289.0 & 281.3 \\
\hline $\operatorname{Poly}(m$-phenylene terephthalamide) & 5.4407 & 271.2 & 280.5 & 272.6 \\
\hline Poly(vinyl $p$ - $t$-butylbenzoate) & 5.3976 & 271.6 & 278.3 & 275.5 \\
\hline Poly ( $p$-phenylene isophthalamide) & 5.4407 & 272.3 & 280.5 & 272.6 \\
\hline Poly(oxy(2,6-diphenyl-1,4-phenylene)) & 5.4033 & 272.8 & 278.6 & 276.5 \\
\hline Poly $(m$-phenylene isophthalamide) & 5.4407 & 280.2 & 280.5 & 272.6 \\
\hline Poly (o-phenylene terephthalamide) & 5.4407 & 286.2 & 280.5 & 272.7 \\
\hline Poly(o-phenylene isophthalamide) & 5.4407 & 291.4 & 280.5 & 272.7 \\
\hline Poly(1,4-cyclohexylidene dimethylene terephthalate) & 6.1826 & 312.3 & 319.3 & 355.6 \\
\hline Poly(11-aminoundecanoic acid) & 5.5116 & 318.3 & 284.3 & 290.2 \\
\hline Poly(di(n-propyl) itaconate) & 5.942 & 328.1 & 306.8 & 311.1 \\
\hline Poly(hexamethylene adipamide) & 6.5148 & 329.2 & 336.7 & 339.1 \\
\hline Poly(12-aminododecanoic acid) & 5.9624 & 331.5 & 307.8 & 314.3 \\
\hline Poly(hexamethylene isophthalamide) & 6.4286 & 344.6 & 332.2 & 330 \\
\hline Poly(hecamethylene azelamide) & 7.8673 & 405.7 & 407.4 & 411.5 \\
\hline Poly(hexamethylene sebacamide) & 8.3182 & 439.0 & 431.0 & 435.6 \\
\hline Nylon-6,12 & 9.2198 & 491.0 & 478.1 & 483.8 \\
\hline overall error* & & & 4.8 & 5.0 \\
\hline
\end{tabular}

which are $x_{\mathrm{C}}=2.92, x_{\mathrm{N}}=7.08, x_{\mathrm{S}}=-0.04, x_{\mathrm{O}}=13.75$, $x_{\mathrm{Cl}}=5.63, x_{\mathrm{Br}}=9.10, x_{\mathrm{F}}=11.82$, and $x_{\mathrm{Si}}=-2.66$ for the atoms of carbon, nitrogen, sulphur, oxygen, chlorine, bromine, fluorine, and silicon in acyclic structure, respectively. And the optimal weights for the atoms of carbon, nitrogen, and oxygen in cyclic structure are $x_{\mathrm{CC}}$ $=9.89, x_{\mathrm{CN}}=0.44$, and $x_{\mathrm{CO}}=1.50$, respectively.

The calculated results with eq 8 and the optimal weights obtained are listed in Table I and depicted in Figure 1. The calculated results of Bicerano are also shown in Table I for comparison. Obviously, the new model shows comparable accuracy with eq 4 , however, the new correlation is more simple, which does not require atomic correction term as well as the $N_{\text {rot }}$ term. By introducing variable weights into the non-hydrogen atoms, the contribution of a certain atom to a property can be increased or decreased, which makes the traditional "fixed-value" connectivity index more flexible, and leads to better correlative accuracy and simpler expression. Once the weights are obtained, the correlations based on the variable connectivity index have the same predictive capability as those based on the traditional connectivity index.

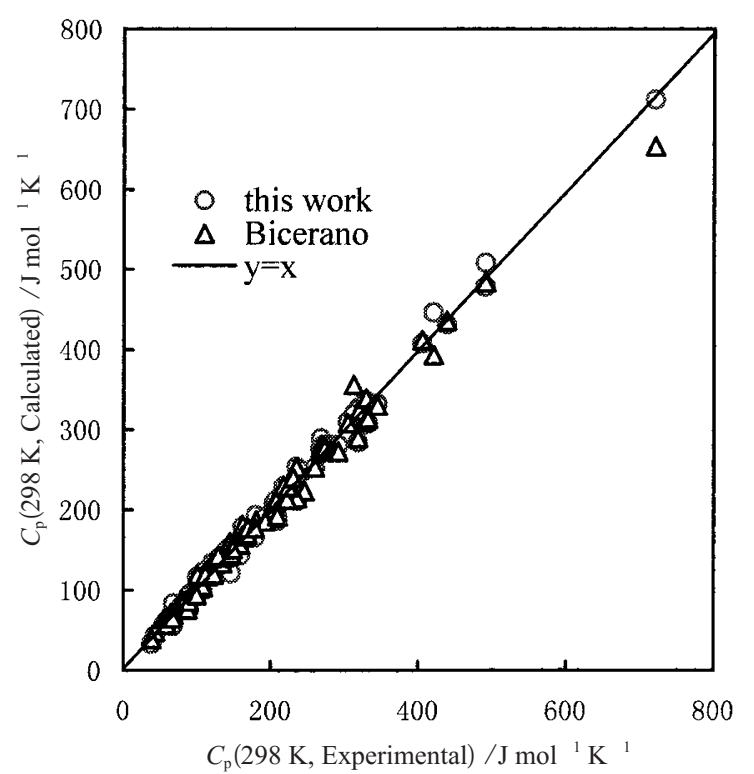

Figure 1. Calculated vs. experimental heat capacity at constant pressure at room temperature for solid polymers.

Correlation of $C_{p}^{l}(298 K)$ for Liquid Polymers

Based on the experimental $C_{\mathrm{p}}^{\mathrm{l}}(298 \mathrm{~K})$ data $^{1}$ of liquid polymers shown in Table II, the following correlation was proposed:

$$
C_{\mathrm{p}}^{1}(298 \mathrm{~K})=52.981{ }^{1} \chi^{f}+5.1689
$$


Table II. Calculated results for $C_{\mathrm{p}}^{\mathrm{l}}(298 \mathrm{~K})$ of liquid polymers and the variable connectivity indices used

\begin{tabular}{|c|c|c|c|c|}
\hline \multirow[t]{2}{*}{ Polymer } & \multirow[t]{2}{*}{${ }^{1} \chi^{f}$} & \multicolumn{3}{|c|}{$\begin{array}{c}C_{\mathrm{P}}^{\mathrm{l}}(298 \mathrm{~K}) \\
\left(\mathrm{J} \mathrm{mol}^{-1} \mathrm{~K}^{-1}\right)\end{array}$} \\
\hline & & exp. & this work & Bicerano \\
\hline Polyoxymethylene & 0.9891 & 57.6 & 57.6 & 54.4 \\
\hline Polyethylene & 1.0811 & 61.6 & 62.4 & 61.3 \\
\hline Poly(vinyl fluoride) & 1.2591 & 72.7 & 71.9 & 74.2 \\
\hline Poly(vinylidene fluoride) & 1.4172 & 82.9 & 80.3 & 88.7 \\
\hline Polyoxyethylene & 1.5297 & 87.8 & 86.2 & 85.1 \\
\hline Polypropylene & 1.5135 & 88.1 & 85.4 & 94.0 \\
\hline Polytrifluoroethylene & 1.6596 & 93.1 & 93.1 & 101.6 \\
\hline Polytetrafluoroethylene & 1.8550 & 102.6 & 103.4 & 116.1 \\
\hline Poly(1,4-butadiene) & 2.1622 & 105.7 & 119.7 & 108.2 \\
\hline Polyisobutylene & 1.8550 & 109.6 & 103.4 & 128.3 \\
\hline Poly(propylene oxide) & 1.9710 & 110.9 & 109.6 & 117.7 \\
\hline Poly(glycolic acid) & 1.8670 & 112.2 & 104.1 & 96.8 \\
\hline Poly(1-butene) & 2.1040 & 116.7 & 116.6 & 121.5 \\
\hline Poly(dimethyl siloxane) & 2.1272 & 117.8 & 117.9 & 121.3 \\
\hline Polyoxytrimethylene & 2.0702 & 119.2 & 114.9 & 115.7 \\
\hline Polyisoprene & 2.5946 & 129.8 & 142.6 & 142.0 \\
\hline Poly(vinylidene chloride) & 2.3610 & 130.0 & 130.3 & 123.8 \\
\hline Poly $(\beta$-propiolactone $)$ & 2.0091 & 135.1 & 111.6 & 127.5 \\
\hline Poly(oxymethyleneoxyethylene) & 2.5188 & 135.9 & 138.6 & 139.4 \\
\hline Poly(oxy $(p$-phenylene $)$ & 2.6020 & 141.6 & 143.0 & 141.5 \\
\hline Polyoxytetramethylene & 2.6107 & 142.8 & 143.5 & 146.4 \\
\hline Poly(1-pentene) & 2.6445 & 143.8 & 145.3 & 149.1 \\
\hline Poly(methyl acrylate) & 2.8885 & 151.7 & 158.2 & 153.9 \\
\hline Poly $(\gamma$-butyrolactone $)$ & 2.9481 & 155.7 & 161.4 & 158.2 \\
\hline Poly(vinyl acetate) & 2.8489 & 156.3 & 156.1 & 153.9 \\
\hline $\operatorname{Poly}($ thio $(p$-phenylene $))$ & 2.8714 & 157.2 & 157.3 & 160.5 \\
\hline Poly(2-methyl-1,4-pentadiene) & 3.1351 & 157.5 & 171.3 & 172.7 \\
\hline Poly(oxy(diethylsilylene)) & 3.2991 & 165.0 & 180.0 & 176.4 \\
\hline Polystyrene & 3.1874 & 174.2 & 174.0 & 174.8 \\
\hline Poly(1-hexene) & 3.1850 & 178.0 & 173.9 & 176.6 \\
\hline Poly(ethyl acrylate) & 3.4509 & 178.5 & 188.0 & 181.4 \\
\hline $\operatorname{Poly}(\delta$-valerolactone $)$ & 3.4886 & 183.2 & 190.0 & 188.8 \\
\hline Poly(methyl methacrylate) & 3.2707 & 183.7 & 178.5 & 188.2 \\
\hline Poly(oxymethyleneoxytetramethylene) & 3.5999 & 194.2 & 195.9 & 200.8 \\
\hline $\operatorname{Poly}(p$-chloro styrene $)$ & 3.8956 & 195.3 & 211.6 & 206.4 \\
\hline Poly( $p$-methyl styrene) & 3.6423 & 197.1 & 198.1 & 208.7 \\
\hline $\operatorname{Poly}(p$-bromo styrene $)$ & 3.6297 & 197.5 & 197.5 & 225.7 \\
\hline Poly(ethyl methacrylate) & 3.5761 & 203.3 & 194.6 & 215.7 \\
\hline Poly( $\varepsilon$-caprolactone $)$ & 4.0292 & 206.5 & 218.6 & 219.5 \\
\hline Poly(oxy(2,6-dimethyl-1,4-phenylene)) & 3.5256 & 208.7 & 192.0 & 209.3 \\
\hline Poly(isobutyl acrylate) & 4.2338 & 231.5 & 229.5 & 241.6 \\
\hline Poly(n-butyl acrylate) & 4.5319 & 232.7 & 245.3 & 236.5 \\
\hline Polyoxynaphthoate & 4.9697 & 239.8 & 268.5 & 255.0 \\
\hline Poly(vinyl benzoate) & 4.5228 & 243.1 & 244.8 & 234.7 \\
\hline Poly(n-butyl methacrylate) & 4.6572 & 262.5 & 251.9 & 270.8 \\
\hline $\operatorname{Poly}(\varepsilon$-caprolactam $)$ & 4.3284 & 269.5 & 234.5 & 221.6 \\
\hline Poly(isobutyl methacrylate) & 4.7561 & 273.5 & 257.2 & 275.9 \\
\hline Poly(vinyl $p$-ethylbenzoate) & 5.5968 & 281.1 & 301.7 & 296.1 \\
\hline Poly(dimethyl itaconate) & 5.1946 & 281.6 & 280.4 & 275.6 \\
\hline Poly(ethylene- $n$-( $\beta$-trimethylsilyl ethyl)imine) & 5.0831 & 286.4 & 274.5 & 271.2 \\
\hline Poly(trimethylene succinate) & 5.3557 & 288.0 & 288.9 & 285.6 \\
\hline Poly(vinyl $p$-isopropylbenzoate) & 6.0114 & 309.4 & 323.7 & 328.8 \\
\hline Poly(ethylene terephthalate) & 5.9236 & 321.2 & 319.0 & 311.5 \\
\hline $\operatorname{Poly}(n$-hexyl methacrylate) & 5.1978 & 324.0 & 280.6 & 325.9 \\
\hline
\end{tabular}




\begin{tabular}{lrrrr} 
Poly(vinylene diphenylsilylene) & 6.0763 & 330.4 & 327.1 & 306.1 \\
Poly(oxy(2,6-diphenyl-1,4-phenylene) & 6.9676 & 337.2 & 374.3 & 370.9 \\
Poly(trimethylene adipate) & 6.4367 & 343.7 & 346.2 & 347.0 \\
Poly(vinyl p-t-butylbenzoate) & 6.3426 & 347.4 & 341.2 & 363.1 \\
Poly(tetramethylene terephthalate) & 7.0047 & 354.5 & 376.3 & 372.8 \\
Polyundecanolactone & 6.7319 & 360.7 & 361.8 & 372.8 \\
Poly(11-aminoundecanoic acid) & 7.0311 & 376.7 & 377.7 & 374.9 \\
Poly(di(n-propyl) itaconate) & 7.4005 & 388.8 & 397.3 & 385.8 \\
Poly(ethylene-2,6-naphthalenedicarboxylate) & 7.3953 & 390.4 & 397.0 & 382.5 \\
Poly(tetramethylene adipate) & 6.9773 & 394.0 & 374.8 & 377.6 \\
Poly(12-aminododecanoic acid) & 7.5716 & 406.4 & 406.3 & 405.6 \\
Bisphenol-A polycarbonate & 7.4787 & 410.8 & 401.4 & 423.1 \\
Polytridecanolactone & 7.8130 & 433.9 & 419.1 & 434.1 \\
Poly(ethylene sebacate) & 8.0584 & 438.3 & 432.1 & 439.0 \\
Poly(ether ether ketone) & 8.2626 & 455.9 & 442.9 & 443.3 \\
Poly(hexamethylene adipamide) & 8.6567 & 490.7 & 463.8 & 443.2 \\
Polypentadecanolactone & 8.8940 & 505.1 & 476.4 & 495.5 \\
Poly(dodecyl methyacrylate) & 9.2385 & 511.5 & 494.6 & 491.1 \\
Poly(hexamethylene sebacate) & 10.2205 & 534.3 & 546.7 & 561.6 \\
Poly(hexamethylene azelamide) & 10.2783 & 545.8 & 549.7 & 535.2 \\
Poly(hexamethylene sebacamide) & 10.8189 & 575.5 & 578.4 & 565.9 \\
Nylon-6,12 & 11.8999 & 634.8 & 635.6 & 627.2 \\
Poly(dicyclooctyl itaconate) & 9.8586 & 663.6 & 527.5 & 602.5 \\
Poly(octadecyl methacrylate) & 12.4817 & 677.8 & 666.5 & 656.3 \\
Udel & 12.2628 & 678.1 & 654.9 & 678.4 \\
\hline Overall error & & & 3.8 & 4.3 \\
\hline
\end{tabular}

The optimal weights for the non-hydrogen atoms in the calculation of ${ }^{1} \chi^{f}$ were obtained by fitting the data, which are $x_{\mathrm{C}}=-0.15, x_{\mathrm{N}}=-0.76, x_{\mathrm{S}}=-0.86, x_{\mathrm{O}}=$ $0.21, x_{\mathrm{Cl}}=-0.60, x_{\mathrm{Br}}=-0.11, x_{\mathrm{F}}=1.33$, and $x_{\mathrm{Si}}=$ -1.27 for the atoms of carbon, nitrogen, sulphur, oxygen, chlorine, bromine, fluorine, and silicon in acyclic structure, respectively. And the optimal weight for carbon atom in cyclic structure is $x_{\mathrm{CC}}=0.84$.

The calculated results and those from eq 5 are shown in Table II and depicted in Figure 2. Similar to that for solid polymers, the new correlation gives comparable accuracy with eq 5 based on the traditional connectivity index, which again illustrates that the utilization of the variable connectivity index can improve correlations either by giving higher accuracy or a simpler expression.

\section{Prediction of the Change in the Heat Capacity at the Glass Transition}

The change in the heat capacity at the glass transition, $\Delta C_{\mathrm{p}}\left(T_{\mathrm{g}}\right)$, is an important property, it can be calculated readily using eqs 6 and 7 combined with the correlations for $C_{\mathrm{p}}^{\mathrm{S}}(298 \mathrm{~K})$ and $C_{\mathrm{p}}^{\mathrm{l}}(298 \mathrm{~K})$ proposed. However, the calculation requires the glass transition temperature values. The experimental $\Delta C_{\mathrm{p}}\left(T_{\mathrm{g}}\right)$ and the corresponding $T_{\mathrm{g}}$ data, as shown in Table III, were taken from the book of Bicerano, ${ }^{1}$ they were employed to test the predictive accuracy of the proposed correlations combined with eqs 6 and 7 for $\Delta C_{\mathrm{p}}\left(T_{\mathrm{g}}\right)$. The

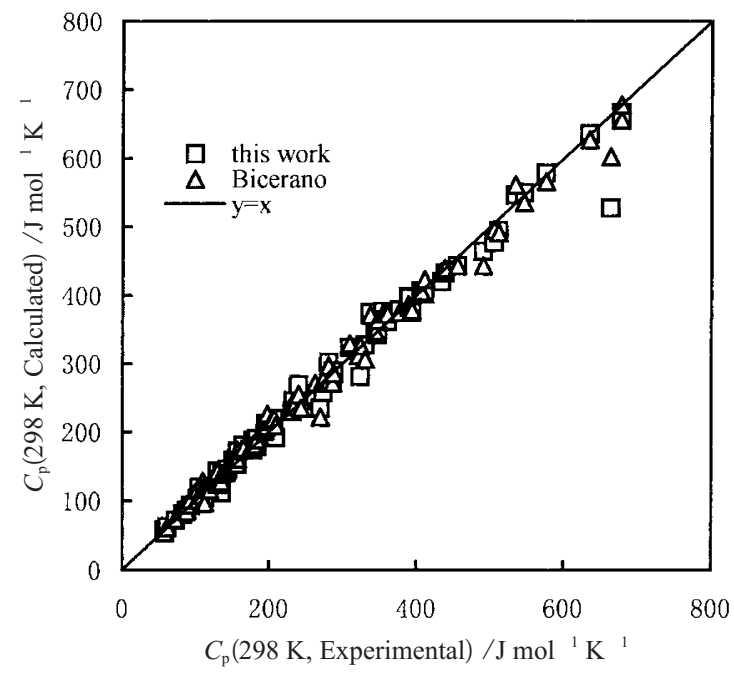

Figure 2. Calculated $v$ s. experimental heat capacity at constant pressure at room temperature for liquid polymers.

predictive results are shown in Table III and depicted in Figure 3. Obviously, the deviation is large, this is expectable because the extrapolation of eqs 6 and 7 to $T_{\mathrm{g}}$ may introduce large error, and the $T_{\mathrm{g}}$ value is not only polymer-dependent but also depends largely on the detailed structure of the polymer concerned. In addition, the experimental data of $\Delta C_{\mathrm{p}}\left(T_{\mathrm{g}}\right)$ may also have large uncertainty. 
Table III. Predicted results for $\Delta C_{\mathrm{p}}\left(T_{\mathrm{g}}\right)$ of polymers

\begin{tabular}{|c|c|c|c|}
\hline \multirow[t]{2}{*}{ Polymer } & \multirow{2}{*}{$\begin{array}{c}T_{\mathrm{g}} \\
(\mathrm{K})\end{array}$} & \multicolumn{2}{|c|}{$\begin{array}{c}\Delta C_{\mathrm{P}}\left(T_{\mathrm{g}}\right) \\
\left(\mathrm{J} \mathrm{mol}^{-1} \mathrm{~K}^{-1}\right)\end{array}$} \\
\hline & & exp. & $\overline{\text { predicted }}$ \\
\hline Polyethylene & 195 & 10.5 & 24.2 \\
\hline Polypropylene & 233 & 19.2 & 23.9 \\
\hline Poly(vinyl chloride) & 348 & 19.4 & 36.9 \\
\hline Poly(vinylidene fluoride) & 233 & 21.2 & 18.2 \\
\hline Polyisobutylene & 199 & 21.3 & 25.2 \\
\hline $\operatorname{Poly}($ oxy $(p$-phenylene $))$ & 358 & 21.4 & 37.4 \\
\hline Poly(1-butene) & 228 & 23.1 & 34.1 \\
\hline Poly(1-hexene) & 223 & 25.1 & 49.9 \\
\hline $\operatorname{Poly}(\alpha$-methyl styrene $)$ & 409 & 26.3 & 18.9 \\
\hline Poly(1-pentene) & 223 & 27.0 & 42.3 \\
\hline Poly(1,4-butadiene $)$ & 171 & 27.2 & 44.0 \\
\hline Poly(dimethyl siloxane) & 152 & 27.7 & 35.3 \\
\hline Polyoxymethylene & 218 & 28.2 & 26.7 \\
\hline Poly(oxy(diethylsilylene)) & 130 & 29.2 & 64.2 \\
\hline Poly(thio ( $p$-phenylene $))$ & 360 & 29.2 & 23.9 \\
\hline Poly(n-butyl methacrylate) & 293 & 29.7 & 55.7 \\
\hline Polystyrene & 373 & 30.8 & 29.9 \\
\hline Polyisoprene & 203 & 30.9 & 43.0 \\
\hline Poly ( $p$-chloro styrene) & 389 & 31.1 & 44.2 \\
\hline Poly(ethyl methacrylate) & 324 & 31.7 & 24.2 \\
\hline Poly(oxy(2,6-dimethyl-1,4-phenylene)) & 482 & 31.9 & 25.7 \\
\hline Poly( $p$-bromo styrene $)$ & 417 & 31.9 & 28.1 \\
\hline Poly(methyl methacrylate) & 378 & 32.7 & 22.8 \\
\hline Poly(propylene oxide) & 206 & 33.2 & 38.1 \\
\hline Poly( $p$-fluoro styrene) & 379 & 33.3 & 25.4 \\
\hline Poly(4-methyl-1-pentene) & 302 & 33.7 & 25.8 \\
\hline Poly( $p$-hydroxybenzoate) & 420 & 34.0 & 41.0 \\
\hline Poly( $p$-methyl styrene) & 374 & 34.6 & 24.6 \\
\hline Poly(isobutyl acrylate) & 249 & 36.6 & 55.2 \\
\hline Poly( $p$-xylylene $)$ & 333 & 37.6 & 36.1 \\
\hline Polyoxyethylene & 206 & 38.2 & 35.1 \\
\hline Poly(isobutyl methacrylate) & 321 & 39.0 & 38.3 \\
\hline Poly(methyl acrylate) & 281 & 42.3 & 44.9 \\
\hline $\operatorname{Poly}(n$-butyl acrylate $)$ & 219 & 45.4 & 77.9 \\
\hline Poly(ethyl acrylate) & 251 & 45.6 & 56.8 \\
\hline Polyoxytrimethylene & 195 & 46.6 & 44.3 \\
\hline Poly(vinyl acetate) & 301 & 46.7 & 39.9 \\
\hline Bisphenol-A polycarbonate & 423 & 48.8 & 40.8 \\
\hline Polyoxytetramethylene & 190 & 52.0 & 53.4 \\
\hline Poly( $\varepsilon$-caprolactone $)$ & 213 & 59.5 & 73.7 \\
\hline Poly(12-aminododecanoicacid) & 310 & 74.3 & 93.9 \\
\hline Poly(11-aminoundecanoic acid) & 315 & 68.4 & 87.5 \\
\hline Poly(oxy(2,6-diphenyl-1,4-phenylene)) & 493 & 76.6 & 27.8 \\
\hline Poly(ethylene terephthalate) & 345 & 77.8 & 76.8 \\
\hline Poly(oxy-1,4-phenylene-oxy-1,4-phenylene-carbonyl-1,4-phenylene) & 419 & 78.1 & 67.2 \\
\hline Poly(ethylene-2,6-naphthalenedicarboxylate) & 397 & 81.6 & 73.4 \\
\hline $\operatorname{Poly}(\varepsilon$-caprolactam $)$ & 335 & 93.6 & 61.0 \\
\hline Polyoxyoctamethylene & 203 & 95.7 & 84.8 \\
\hline Poly(hexamethylene sebacamide) & 313 & 118.0 & 139.5 \\
\hline Poly(tetramethylene adipate) & 205 & 140.0 & 126.9 \\
\hline Poly(hexamethylene adipamide) & 330 & 145.0 & 114.3 \\
\hline Poly(ethylene sebacate) & 243 & 154.0 & 127.2 \\
\hline Overall error & & & 32.2 \\
\hline
\end{tabular}




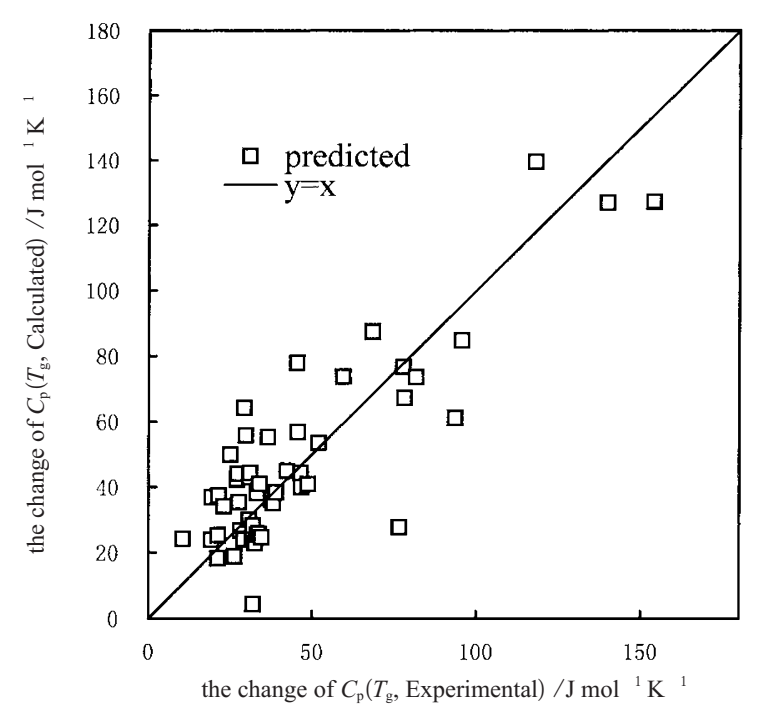

Figure 3. Predicted $v s$. experimental the change in the heat capacity at the glass transition.

\section{CONCLUSION}

Two correlations based on the variable connectivity index were proposed for the heat capacity at constant pressure for solid and liquid polymers, respectively. The correlations are simple with comparable correlative accuracy to the existing ones. The present work shows that the variable connectivity index has more flexibility in characterizing polymers, which can lead to simpler correlations with better correlative accuracy.

Acknowledgment. The financial support of the Ministry of Education of China (Contract: 99013) is greatly appreciated. And the authors are also grateful for the valuable comments of Dr. J. Bicerano of the Dow Chemical Company on the manuscript.

\section{LIST OF SYMBOLS}

$C_{\mathrm{p}}(T), \quad$ Heat capacity at constant pressure $\left[\mathrm{J} \mathrm{mol}^{-1} \mathrm{~K}^{-1}\right.$ ]

$C_{\mathrm{p}}(298 \mathrm{~K})$, Heat capacity at constant pressure at room temperature $\left[\mathrm{J} \mathrm{mol}^{-1} \mathrm{~K}^{-1}\right]$

$\Delta C_{\mathrm{p}}\left(T_{\mathrm{g}}\right), \quad$ The change in the heat capacity at the glass transition $\left[\mathrm{J} \mathrm{mol}^{-1} \mathrm{~K}^{-1}\right]$

$N, \quad$ The number of data points

$N_{\text {BBrot}}, \quad$ The rotational degrees of freedom of the backbone portion of the repeat units of polymer

$N_{\text {rot }}, \quad$ The total number of rotational degrees of freedom
$N_{\text {SGrot}}, \quad$ The rotational degrees of freedom of the side group portion of the repeat units of polymer

$n_{m}, \quad$ The number of the relevant paths

$N_{\text {si }}, \quad$ The number of silicon atoms

$T, \quad$ Temperature $[\mathrm{K}]$

$T_{\mathrm{g}}, \quad$ Glass transition temperature of polymers

[K]

$x_{\mathrm{i}}, \quad$ The weight to a non-hydrogen atom

Greek letters

${ }^{m} \chi$, $\quad$ mth-order connectivity index

${ }^{m} \chi_{t}{ }^{f}, \quad$ mth-order variable connectivity index

${ }^{m} \chi_{t}{ }^{v}, \quad$ mth-order valence connectivity index

$\delta, \quad$ The simple connectivity index

$\delta^{\mathrm{v}}, \quad$ The valence connectivity index

$\delta^{\mathrm{f}}, \quad$ The variable connectivity index

Subscripts

i,

t,

Superscripts

cal., $\quad$ Calculated value

exp., Experimental value

1, $\quad$ Liquid

S, Solid

\section{REFERENCES}

1. J. Bicerano, "Prediction of Polymer Properties", 3rd ed., Marcel Dekker, Inc., New York, N.Y., 2002.

2. D. W. Van Krevelen, "Properties of Polymers. Their Correlation with Chemical Structure, Their Numerical Estimation and Prediction from Additive Group Contributions", 3rd ed., Elsevier Science Publishers, Amsterdam, 1990.

3. C. R. Novenario, J. M. Caruthers, and K.-C. Chao, J. Appl. Polym. Sci., 67, 841 (1998).

4. F. Wang, S. Saeki, and T. Yamaguchi, Polymer, 39, 5139 (1998).

5. M. Pyda, Macromolecules, 35, 4009 (2002).

6. C. Zhong and C. Yang, J. Polym. Sci., Polym. Phys. Ed., 40, 401 (2002).

7. C. Zhong, Q. Li, and C. Yang, Ind. Eng. Chem. Res., 41, 2826 (2002).

8. M. Randić, J. Comput. Chem., 12, 970 (1991).

9. L. B. Kier and L. H. Hall, "Molecular Connectivity in Chemistry and Drug Research", Academic Press, Inc., New York, N.Y., 1976.

10. L. B. Kier and L. H. Hall, "Molecular Connectivity in Structure-Activity Analysis", John Wiley \& Sons Inc., New York, 1986.

11. M. Randić, J. Mol. Graphics Modell, 20, 19 (2001).

12. M. Randić, D. Plavsic, and N. Lers, J. Chem. Inf. Comput. Sci., 41, 657 (2001). 